\title{
Correlation between DTI effect and white matter structural integrity, working memory in leukoaraiosis patients
}

\author{
XIAOFENG YANG ${ }^{1}$, WANMENG XIE ${ }^{1 *}$ \\ ${ }^{1}$ Department of Medical Imaging, Suizhou Hospital Hubei University of Medicine (Suizhou \\ Central Hospital), Suizhou, 441300, PR China
}

\begin{abstract}
Our objective was to study the correlation between Diffusion tensor MR imaging (DTI) effect and white matter structural integrity, working memory in leukoaraiosis patients. 100 leukoaraiosis patients referring to the First Affiliated Hospital of Beijing Medical University from December 2018 to December 2019, were selected as study subjects and divided into four groups according to disease severity: lesion-free group, mild lesion group, moderate lesion group, and severe lesion group. All patients underwent magnetic resonance diffusion tensor imaging to collect DWI images and analyze Fractional anisotropy (FA), mean diffusivity (MD), ReHo values of white matter area under different grading. The patients' working memory was tested via auditory verb learning test and Stroop color word test, so that correlation between white matter structural integrity and working memory can be analyzed.

Results: There are statistically significant differences in FA values of the right posterior thalamic radiation, the right sagittal layer and the right superior longitudinal fasciculus, MD values of the right sagittal layer, the right cingulum bundle, the left cingulum bundle, the right inferior fasciculus fronto-occipitalis and the left inferior fasciculus frontooccipitalis, as well as instant recall, delayed recall, delayed recognition, card A (dot), card B (character), card $\mathrm{C}$ (color word) and SIE value $(P<0.01)$. Correlation is shown between white matter structural integrity and working memory, gender, age, grading, disease course, recurrence interval, white matter area, and testing methods. There was a correlation between DTI effect and white matter structural integrity, working memory in leukoaraiosis patients, and leukoaraiosis patients have memory impairment.
\end{abstract}

Keywords Leukoaraiosis patients, DTI effect, white matter structural integrity, working memory, correlation.

To cite this article: YANG X, XIE W. Correlation between DTI effect and white matter structural integrity, working memory in leukoaraiosis patients. Rom Biotechnol Lett. 2021; 26(3): 2714-2721. DOI: $10.25083 / \mathrm{rbl} / 26.3 / 2714-2721$

*Corresponding author: WANMENG XIE, Department of Medical Imaging, Suizhou Hospital Hubei University of Medicine (Suizhou Central Hospital), Suizhou, 441300, PR China

E-mail: drcorespond@gmail.com 


\section{Introduction}

Leukoraiosis (LA) is a demyelinating disease of nerve conduction fibers caused by diffuse cerebral ischemia, whose main clinical manifestations are chronic progressive dementia, slow thinking, and cognitive function decline, etc. The disease has aroused concern of more and more specialists in recent years [1-2]. Although clinical MRI is a very sensitive imaging technique to detect abnormalities in the white matter of the brain, it has little feature in accurately detecting the stages of demyelination. On the other hand, routine MRI imaging includes T1 and T2 weighted images with little insight into the white matter status of normal appearance [2]. Usually, white matter MRI lesions are concerned with vascular diseases of brain and are often linked to as leukoaraiosis (LA) [1, 3].

Magnetic resonance diffusion tensor imaging (MR-DTI) is the only imaging method capable of studying abnormalities such as metastasis and destruction of white matter fiber tracts in living tissues [3-4], which is increasingly widely used in the detection of leukoaraiosis patients. This technology can not only provide information about the diseased brain area, but also perform qualitative and quantitative analysis of the disease based on analysis software. With more accurate resolution and effect, it provides patients with the most accurate treatment plan [5-7].

While there are many reports of using DTI to asses and diagnosis Leukoraiosis, little number of papers have looked at the possible correlations of the DTI outputs and disease state, considering the patient memory function.

In this experiment, 100 leukoaraiosis patients were selected as study subjects for magnetic resonance diffusion tensor imaging to study the correlation between DTI effect and white matter structural integrity, working memory in leukoaraiosis patients.

\section{Information and Methods}

Patients with leukoaraiosis confirmed by pathology in the First Affiliated Hospital of Beijing Medical University from December 2018 to December 2019 were selected as study subjects, by simple method of sampling.

Inclusion criteria: [1] All patients meet the diagnostic criteria for leukoaraiosis; [2] All patients have a medical history longer than 5 years; [3] The patients' clinical data are complete and accurate, and there are no other cardiovascular and cerebrovascular diseases.

Exclusion criteria: [1] Patients with leukoencephalopathy, encephalitis, brain trauma, and large-area cerebral infarction [2]. Complicated with other neuropsychiatric diseases such as primary headaches, head trauma, brain tumors, multiple sclerosis, mental disorders, and strokes, or have history of craniocerebral surgery; [3] Combined with severe heart, liver, and kidney, etc. failure or malignant tumors; [4] The patient has contraindications to magnetic resonance imaging. All subjects in this trial have signed informed consent.

According to the disease severity, patients were divided into lesion-free group, mild lesion group, moderate lesion group and severe lesion group. The specific grading standards are: patients with no lesions belong to grade 0 ; patients with mild lesions belong to grade 1, that is, bilateral periventricular white matter has scattered localized lesions; patients with moderate lesions belong to grade 2, that is, bilateral periventricular white matter has non-fusion or partial fusion lesions; patients with severe lesions belong to grade 3 , that is, the lesions fuse and involve all the periventricular white matter [8].

All patients underwent MRI scans. In the examination, the patient took a supine position, and MRI scan of the pituitary gland was performed with a Siemens Magnetom Trio 3.0T superconducting magnetic resonance imaging instrument and an 8-channel coil on the head. Scan parameters: sagittal $\mathrm{T} 1 \mathrm{WI}(\mathrm{TR}=250 \mathrm{~ms}, \mathrm{TE}=2.6 \mathrm{~ms})$, coronal T1WI $(\mathrm{TR}=750 \mathrm{~ms}, \mathrm{TE}=8.1 \mathrm{~ms}), \mathrm{T} 2 \mathrm{WI}$ $(\mathrm{TR}=4000 \mathrm{~ms}, \mathrm{TE}=83 \mathrm{~ms}$ ), slice thickness $2 \mathrm{~mm}$, layer spacing $0.2 \mathrm{~mm}$, matrix $320 \times 256$, number of layers 15 , signal acquisition times 3 times. The patient underwent dynamic and conventional enhanced scanning (intravenous bolus injection of contrast agent Gd-DTPA, dosage $0.1 \mathrm{mmoL} / \mathrm{kg}$ ). Dynamic enhanced scanning adopted coronal T1WI: $\mathrm{TR}=160 \mathrm{~ms}, \mathrm{TE}=2.83 \mathrm{~ms}$, slice thickness $2 \mathrm{~mm}$, slice interval $0.2 \mathrm{~mm}$, FOV $200 \mathrm{~mm}$, continuous scanning 6 times, 9 slices each time. After dynamic enhanced scanning, conventional sagittal and coronal enhanced T1WI scanning was performed with scanning parameters the same as those of plain scan to collect DWI images.

\section{FA and MD values of white matter}

Through Functool 1l DTI, the obtained magnetic resonance image was post-processed to reconstruct FA image and three-dimensional color-coded tensor image. FA is an index for the sum of diffusion asymmetry within a voxel. After FA image reconstruction, the relevant FA values were measured for the right posterior thalamic radiation, right sagittal layer, and right superior longitudinal fasciculus; at the same time, MD values were measured for the right sagittal layer, right cingulum bundle, left cingulum bundle, right inferior fasciculus fronto-occipitalis and the left inferior fasciculus frontooccipitalis. ROI area in all measurements was about 25 pixels. The average magnitude of molecular displacement by diffusion represents MD.

\section{ReHo value analysis on the white matter area image}

In accordance with the method 1.2, plain scan and enhanced MRI scan of the lesion and adjacent parts were performed using MRI scanner. The scan sequences included spin rotation and fast spin rotation, with scan 
thickness $2 \mathrm{~mm}$, interval $2 \mathrm{~mm}$ and time interval $120 \mathrm{~ms}$. MRI images of all patients were analyzed to calculate the ReHo value and analyze the correlation of the patient's local brain activity.

\section{Auditory verb learning tests}

The Rey Auditory Verbal Learning Test assess the extent and severity of memory dysfunction and, over time, monitor improvements in memory function [9].

Working memory tests for patients of different grading mainly include immediate recall, delayed recall and delayed recognition. The test method is as follows: [1] The tester reads 15 common vocabularies in daily life (group A) at a rate of one word per second, and the testee is required to repeat the words immediately after listening to 15 words, three times in a row. Each repeat time is $2 \mathrm{~min}$, and the average of the three repeat scores is taken as the immediate recall score; [2] After $15 \mathrm{~min}$, the testee is required to recall and repeat 15 words of group A still in $2 \mathrm{~min}$, and the recall score is delayed recall score; [3] After the second step is completed, tell the testee that the next 30 words will be read out, including 15 words from group A, and 15 new common words (group B). The time is still $2 \mathrm{~min}$, and the score of correct answer minus that of wrong answer is the delayed recognition score.

\section{Stroop color word tests}

The Stroop Color and Word Test (SCWT) is a psychological model which is commonly used to evaluate the ability to suppress cognitive interference which arises whenever the processing of a particular stimulus feature prevents a second stimulus feature from being processed simultaneously, known as the Stroop Effect [10].

The test material is 3 cards in 4 colors of red, green, yellow, and blue, and each card has 24 dots or characters [1]. Present card A (dot) composed of 24 dots in the above 4 different colors, require the testee to read the dot color quickly and accurately, and record the time; [2] Present card B (character) composed of 4 common Chinese characters in 4 colors, require the testee to read out the color of Chinese characters quickly and accurately, and record the time; [3] Present card C (color word) composed of 4 Chinese characters indicating color, require the testee to read the word color quickly and accurately, rather than meaning of the word itself, and record the time. Interference effect $(\mathrm{SIE})=$ time consumption of card C-time consumption of card B.

\section{Statistical methods}

To assess the correlation between white matter structural integrity and working memory, one-way analysis of variance was used for comparison between multiple samples, and Spearman correlation analysis method was taken to improve comparative correlation of sample mean between the SNK-q test groups. $P<0.05$ indicates difference in the data between the groups, and $P<0.01$ indicates significant difference. A comparative analysis was made on the above-mentioned clinical data of the experiment participants, mainly including age, gender, disease course and underlying diseases (whether there is medical history of diabetes, hypertension, heart disease, etc.). The experimental data were counted and analyzed by SPSS 20.0 software. The experimental data were expressed as mean \pm standard deviation $(\mathrm{x} \pm \mathrm{s})$. One-way analysis of variance was used to analyze significance of difference in data between the groups. $P<0.05$ indicates data differences between groups, $P<0.01$ indicates significant difference.

\section{Results}

In this study, 100 patients were divided into lesionfree group $(n=25)$, mild lesion group $(n=25)$, moderate lesion group $(n=25)$ and severe lesion group $(n=25)$. There were 68 males and 32 females, with an average age of (65.24 \pm 6.18$)$ and a disease course of 5-10 years.

There was no difference in age, gender, disease course and underlying disease between study groups (whether there is history of diabetes, hypertension, heart disease) between lesion-free group, mild lesion group, moderate lesion group, and severe lesion group $(P>0.05)$ (as shown in Table 1).

Table 1. Comparative analysis of patients' general data

\begin{tabular}{lcccccc}
\hline Item & $\begin{array}{c}\text { lesion-free group } \\
(\mathrm{n}=25)\end{array}$ & $\begin{array}{c}\text { mild lesion group } \\
(\mathrm{n}=25)\end{array}$ & $\begin{array}{c}\text { moderate lesion } \\
\text { group }(\mathrm{n}=25)\end{array}$ & $\begin{array}{c}\text { severe lesion } \\
\text { group }(\mathrm{n}=25)\end{array}$ & $F$ value & $P$ value \\
\hline Age (years) & $65.14 \pm 4.32$ & $67.12 \pm 7.01$ & $62.56 \pm 4.09$ & $64.44 \pm 3.21$ & 2.341 & 0.124 \\
\hline Gender (male/female) & $12 / 13$ & $15 / 10$ & $11 / 14$ & $14 / 11$ & 2.563 & 0.221 \\
\hline Disease course (years) & $7.78 \pm 0.78$ & $7.45 \pm 1.22$ & $7.98 \pm 0.97$ & $7.21 \pm 1.01$ & 6.114 & 0.001 \\
\hline Hypertension (n, \%) & $1(4.00)$ & $1(4.00)$ & $3(12.00)$ & $5(20.00)$ & 1.223 & 0.605 \\
\hline Diabetes (n, \%) & $3(12.00)$ & $2(8.00)$ & $5(20.00)$ & $8(32.00)$ & 0.789 & 0.702 \\
\hline Heart disease (n, \%) & $0(0.00)$ & $0(0.00)$ & $1(4.00)$ & $5(20.00)$ & 2.347 & 0.107 \\
\hline Other diseases (n, \%) & $0(0.00)$ & $0(0.00)$ & $1(4.00)$ & $3(12.00)$ & 3.541 & 0.644 \\
\hline
\end{tabular}


There are statistically significant differences in FA values of the right posterior thalamic radiation, the right sagittal layer and the right superior longitudinal fasciculus, MD values of the right sagittal layer, the right cingulum bundle, the left cingulum bundle, the right inferior fasciculus fronto-occipitalis and the left inferior fasciculus fronto-occipitalis $(P<0.01)$. FA values of the right posterior thalamic radiation, the right sagittal layer, and the right superior longitudinal fasciculus are lower in the lesion-free group than in the other three groups. MD values of the right sagittal layer, the right cingulum bundle, the left cingulum bundle, the right inferior fasciculus frontooccipitalis and the left inferior fasciculus fronto-occipitalis are higher in the lesion-free group than in the other three groups (as shown in Table 2).

Table 2. Comparative analysis of FA and MD values of white matter areas in patients of different grading

\begin{tabular}{|c|c|c|c|c|c|c|c|c|}
\hline \multirow[t]{2}{*}{ Group } & \multicolumn{3}{|c|}{ FA value } & \multicolumn{5}{|c|}{ MD value $\left(\times 10^{-3} \mathrm{~mm}^{2} / \mathrm{s}\right)$} \\
\hline & $\begin{array}{l}\text { the right } \\
\text { posterior } \\
\text { thalamic } \\
\text { radiation }\end{array}$ & $\begin{array}{c}\text { the right } \\
\text { sagittal layer }\end{array}$ & $\begin{array}{l}\text { the right } \\
\text { superior } \\
\text { longitudinal } \\
\text { fasciculus }\end{array}$ & $\begin{array}{c}\text { the right } \\
\text { sagittal layer }\end{array}$ & $\begin{array}{l}\text { the right } \\
\text { cingulum } \\
\text { bundle }\end{array}$ & $\begin{array}{l}\text { the left } \\
\text { cingulum } \\
\text { bundle }\end{array}$ & $\begin{array}{l}\text { the right } \\
\text { inferior } \\
\text { fasciculus } \\
\text { fronto- } \\
\text { occipitalis }\end{array}$ & $\begin{array}{l}\text { the left } \\
\text { inferior } \\
\text { fasciculus } \\
\text { fronto- } \\
\text { occipitalis }\end{array}$ \\
\hline $\begin{array}{l}\text { lesion-free } \\
\text { group }(n=25)\end{array}$ & $0.52 \pm 0.01$ & $0.51 \pm 0.02$ & $0.54 \pm 0.02$ & $0.82 \pm 0.01$ & $0.73 \pm 0.01$ & $0.70 \pm 0.03$ & $0.79 \pm 0.02$ & $0.78 \pm 0.02$ \\
\hline $\begin{array}{l}\text { Mild lesion } \\
\text { group }(n=25)\end{array}$ & $0.54 \pm 0.02$ & $0.52 \pm 0.01$ & $0.55 \pm 0.01$ & $0.79 \pm 0.05$ & $0.70 \pm 0.04$ & $0.69 \pm 0.02$ & $0.77 \pm 0.04$ & $0.76 \pm 0.04$ \\
\hline $\begin{array}{l}\text { Moderate } \\
\text { lesion group } \\
(\mathrm{n}=25)\end{array}$ & $0.57 \pm 0.01$ & $0.55 \pm 0.06$ & $0.58 \pm 0.05$ & $0.75 \pm 0.04$ & $0.66 \pm 0.01$ & $0.65 \pm 0.05$ & $0.76 \pm 0.01$ & $0.73 \pm 0.01$ \\
\hline $\begin{array}{l}\text { Severe lesion } \\
\text { group }(\mathrm{n}=25)\end{array}$ & $0.59 \pm 0.05$ & $0.57 \pm 0.04$ & $0.60 \pm 0.06$ & $0.74 \pm 0.03$ & $0.63 \pm 0.02$ & $0.63 \pm 0.01$ & $0.72 \pm 0.02$ & $0.70 \pm 0.04$ \\
\hline$F$ value & 6.596 & 6.112 & 11.587 & 9.564 & 13.339 & 6.546 & 6.269 & 6.361 \\
\hline$P$ value & 0.006 & 0.001 & 0.001 & 0.001 & 0.001 & 0.003 & 0.001 & 0.001 \\
\hline
\end{tabular}
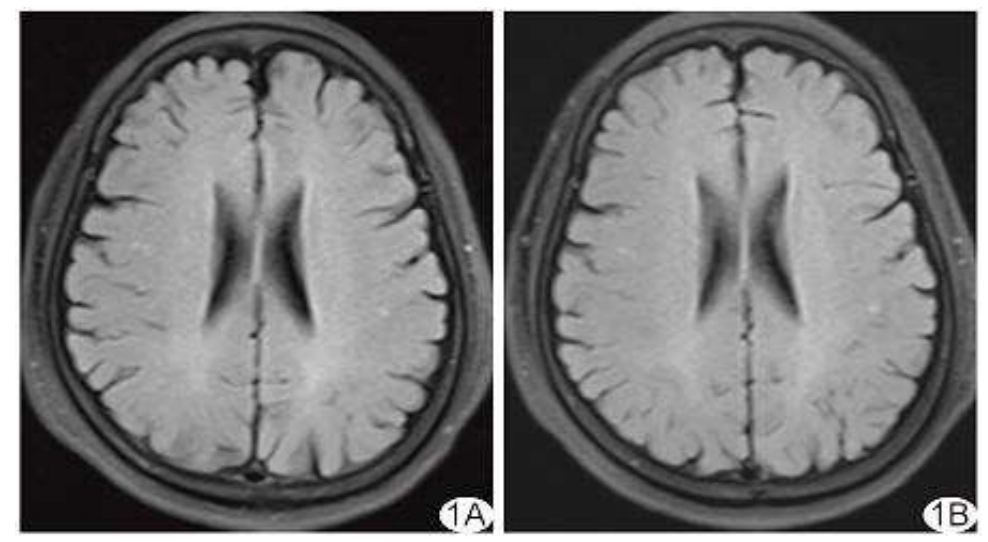

Figure 1A-1B. Magnetic resonance diffusion tensor imaging of the white matter; where, $1 \mathrm{~A}$ represents leukoaraiosis patient, and $1 \mathrm{~B}$ represents healthy people

The standard ReHo image is derived according to the functional magnetic resonance imaging results of the diseased brain area of leukoaraiosis patients, and the relevant data is accessed by ReHo image processing. Where, brain areas with decreased ReHo value in leukoaraiosis patients are the right calcarine gyrus, right middle occipital gyrus, left cuneate convolution, bilateral middle frontal gyrus, anterior cingulate gyrus, right supramarginal gyrus, and posterior central gyrus. The brain areas with increased ReHo values include the left putamen, hippocampus, parahippocampal gyrus, right putamen, caudate nucleus head, hippocampus, left inferior parietal lobule, and left superior frontal gyrus ventral (as shown in Table 3). 
Table 3. ReHo value analysis on the patient's white matter area imaging

\begin{tabular}{lcccc}
\hline \multicolumn{1}{c}{ Brain area } & BA peak & $\begin{array}{l}\text { voxel } \\
\text { value }\end{array}$ & $t$ value & peak MNI coordinates (x, y, z) \\
\hline Brain areas with decreased ReHo value & & & & $24,-57,15$ \\
\hline right calcarine gyrus & 17 & 172 & -5.60 & $30,-93,3$ \\
\hline right middle occipital gyrus & 18 & 118 & -3.88 & $-9,-99,3$ \\
\hline $\begin{array}{l}\text { left cuneate convolution } \\
\text { bilateral middle frontal gyrus, anterior } \\
\text { cingulate gyrus }\end{array}$ & 18 & 129 & -4.25 & $-3,45,-6$ \\
\hline $\begin{array}{l}\text { right supramarginal gyrus, posterior } \\
\text { central gyrus }\end{array}$ & 2 & 196 & -3.88 & $57,-30,33$ \\
\hline $\begin{array}{l}\text { brain areas with increased ReHo value } \\
\text { left putamen, hippocampus, } \\
\text { parahippocampal gyrus }\end{array}$ & 122 & -5.73 & $-30,-9,-9$ \\
\hline $\begin{array}{l}\text { right putamen, caudate nucleus head, } \\
\text { hippocampus }\end{array}$ & 48 & 90 & 3.58 & $39,9,15$ \\
\hline $\begin{array}{l}\text { left inferior parietal lobule } \\
\text { left superior frontal gyrus ventral }\end{array}$ & 60 & 107 & 4.51 & $-21,-48,39$ \\
\hline
\end{tabular}

Note: $t>0$ means leukoarais patient has higher value than the control group, and $t>0$ means leukoarais patient has lower value than the control group; the peak BA represents Brodmann brain area.

There are statistically significant differences in auditory verb learning test results between patients of different grading $(P<0.01)$. Where, immediate recall, delayed recall and delayed recognition of the lesion-free group are scored $(6.26 \pm 0.98),(7.76 \pm 1.02)$, and $(8.41 \pm 1.45)$, respectively; those of the mild lesion group are scored $(6.19 \pm 1.03),(7.60 \pm 0.87)$, and $(5.97 \pm 0.93)$, respectively; those of the moderate lesion group are scored (5.95 \pm 1.21$),(7.33 \pm 0.99)$, and $(5.61 \pm 1.04)$, respectively; those of the severe lesion group are scored $(5.03 \pm 0.81)$, $(6.74 \pm 0.76)$ and $(5.11 \pm 0.45)$, respectively.

Table 4. Comparative analysis on auditory verb learning tests in patients of different grading

\begin{tabular}{lcccccc}
\hline Item & $\begin{array}{c}\text { lesion-free } \\
\text { group }(\mathrm{n}=25)\end{array}$ & $\begin{array}{c}\text { mild lesion group } \\
(\mathrm{n}=25)\end{array}$ & $\begin{array}{c}\text { moderate lesion } \\
\text { group }(\mathrm{n}=25)\end{array}$ & $\begin{array}{c}\text { severe lesion } \\
\text { group }(\mathrm{n}=25)\end{array}$ & $F$ value & $P$ value \\
\hline $\begin{array}{l}\text { immediate } \\
\text { recall }\end{array}$ & $6.26 \pm 0.98$ & $6.19 \pm 1.03$ & $5.95 \pm 1.21$ & $5.03 \pm 0.81$ & 27.432 & 0.002 \\
\hline delayed recall & $7.76 \pm 1.02$ & $7.60 \pm 0.87$ & $7.33 \pm 0.99$ & $6.74 \pm 0.76$ & 16.311 & 0.001 \\
\hline $\begin{array}{l}\text { delayed } \\
\text { recognition }\end{array}$ & $6.41 \pm 1.45$ & $5.97 \pm 0.93$ & $5.61 \pm 1.04$ & $5.11 \pm 0.45$ & 12.034 & 0.004 \\
\hline
\end{tabular}

There are statistically significant differences in Stroop color word test results between patients of different grading $(\mathrm{P}<0.01)$. Where, card A (dot), card B (character), card $\mathrm{C}$ (color word) and SIE values of the lesion-free group are $(24.56 \pm 3.23),(30.76 \pm 2.98),(40.44 \pm 4.21)$ and $(9.96 \pm 1.21)$, respectively; those of mild lesion group are
(25.76 \pm 2.34$),(32.91 \pm 3.12),(47.87 \pm 4.65)$ and $(15.21 \pm 2.03)$ respectively; those of moderate lesion group are (28.97 \pm 4.54$),(35.09 \pm 1.90),(50.32 \pm 4.44)$ and $(21.75 \pm 3.45)$, respectively; those of severe lesion group are $(31.11 \pm 3.12),(39.65 \pm 3.98),(53.46 \pm 6.12)$ and $(27.54 \pm 2.97)$, respectively.

Table 5. Comparative analysis on Stroop color word tests in patients of different grading

\begin{tabular}{lcccccc}
\hline Item & $\begin{array}{c}\text { lesion-free } \\
\text { group }(\mathrm{n}=25)\end{array}$ & $\begin{array}{c}\text { mild lesion group } \\
(\mathrm{n}=25)\end{array}$ & $\begin{array}{c}\text { moderate lesion } \\
\text { group }(\mathrm{n}=25)\end{array}$ & $\begin{array}{c}\text { severe lesion } \\
\text { group }(\mathrm{n}=25)\end{array}$ & $t$ value & $P$ value \\
\hline card A (dot) & $24.56 \pm 3.23$ & $25.76 \pm 2.34$ & $28.97 \pm 4.54$ & $31.11 \pm 3.12$ & 6.986 & 0.001 \\
\hline card B (character) & $30.76 \pm 2.98$ & $32.91 \pm 3.12$ & $35.09 \pm 1.90$ & $39.65 \pm 3.98$ & 7.234 & 0.005 \\
\hline card C (color word) & $40.44 \pm 4.21$ & $47.87 \pm 4.65$ & $50.32 \pm 4.44$ & $53.46 \pm 6.12$ & 6.990 & 0.001 \\
\hline SIE & $9.96 \pm 1.21$ & $15.21 \pm 2.03$ & $21.75 \pm 3.45$ & $27.54 \pm 2.97$ & 7.112 & 0.001 \\
\hline
\end{tabular}


Multivariate Logistic regression analysis was performed, with white matter structural integrity and working memory as dependent variables. The independent variables are gender, age, grading, disease course, recurrence interval, white matter area and testing methods. The analysis found that, independent influencing factors that affect brain white matter structural integrity and working memory include gender $(\mathrm{OR}=2.013, P<0.05)$, age $(\mathrm{OR}=1.917, P<0.05)$, grading $(\mathrm{OR}=1.968, P<0.05)$, disease course $(\mathrm{OR}=2.042, P<0.05)$, white matter area $(\mathrm{OR}=1.986, P<0.05)$ and testing method $(\mathrm{OR}=1.422$, $P<0.05$ ), (as shown in Table 6).

Table 6. Analysis of multiple factors affecting postoperative recurrence

\begin{tabular}{lcccccc}
\hline \multicolumn{1}{c}{ Factor } & $\beta$ value & SE & $\begin{array}{c}\text { WaldX } \\
\text { value }\end{array}$ & $P$ value & OR value & $95 \%$ CI \\
\hline Gender & 0.065 & 13.499 & 0.981 & 0.011 & 2.013 & $0.361-0.842$ \\
\hline Age (years) & 0.267 & 14.439 & 0.091 & 0.002 & 1.917 & $0.098-0.987$ \\
\hline Grading & 0.443 & 17.598 & 0.109 & 0.005 & 1.968 & $0.281-0.662$ \\
\hline $\begin{array}{l}\text { Disease Course } \\
\text { (years) }\end{array}$ & 0.299 & 13.470 & 0.449 & 0.006 & 2.042 & $0.118-0.287$ \\
\hline $\begin{array}{l}\text { White matter area } \\
\text { Testing method }\end{array}$ & 0.194 & 12.749 & 0.220 & 0.001 & 1.986 & $0.119-0.327$ \\
\hline
\end{tabular}

\section{Discussion}

Leukopenia refers to a group of clinical syndromes described by imaging, which involves various causes. Belonging to one of the most common types of cerebral small vessel disease, it has atypical early clinical symptoms. As the disease progresses, the main clinical manifestations are cognitive dysfunction, abnormal gait and mood changes, etc. [11-13]. In recent years, cognitive dysfunction of leukoaraiosis patients has become a research focus of the disease. Studies have shown [14] that the cognitive impairment areas mainly involve executive function, memory, attention and so on. Different from conventional MRI, magnetic resonance diffusion tensor imaging can detect microscopic motion of water molecules, which is the only method used to detect the trajectory of water molecules in living tissues. In the central nervous system, it is used to study integrity of myelin sheath, which can reveal the disease progression at the microscopic level [15-17]. In this experiment, 100 leukoaraiosis patients were selected as study subjects for magnetic resonance diffusion tensor imaging to collect DWI images and analyze FA, MD, ReHo values of white matter area under different grading. The patients' working memory was tested via auditory verb learning test and Stroop color word test, so that correlation between white matter structural integrity and working memory can be analyzed. The experimental results indicate significant differences between patients of different grading in FA and MD values of the white matter area, immediate recall, delayed recall and delayed recognition, as well as Stroop color word test results $(P<0.01)$. Correlation is shown between white matter structural integrity and working memory, gender, age, grading, disease course, recurrence interval, white matter area, and testing methods. Hence, there is a correlation between DTI effect and white matter structural integrity, working memory in leukoaraiosis patients, and leukoaraiosis patients have memory impairment.

In the study of O'Sullivan et al., it was indicated that in leukoaraiosis patients, In contrast to traditional MRI tests, DTI correspond better with cognition [18]. This finding was confirmed by our study.

Our study showed that there may be a correlation between DTI effect and white matter structural integrity, working memory in leukoaraiosis patients. Zhao et al. conducted a DTI study on leukoaraiosis patients and concluded that DTI has good sensitivity in comparison of the MRI and could be used to assess the early lesions appearing in normal MRI. As well as our study, their study indicated different FA and MD values in different parts of the brain [19].

In our study the highest voxel values happened at right calcarine gyrus. In the study of Zhu et al. LA patients had increased Right Middle temporal gyrus, left Lingual gyrus, Middle occipital gyrus, Anterior cingulate gyrus, left Middle occipital gyrus had the highest voxel value [20].

Finally, their study indicated increased ReHo of right middle frontal gyrus and right anterior cingulate gyrus; While in our study, left putamen, hippocampus, parahippocampal gyrus, right putamen, caudate nucleus head, hippocampus, left inferior parietal lobule, and left superior frontal gyrus ventral had increased ReHo. 
Clinical studies have shown that magnetic resonance diffusion tensor imaging (MR-DTI) can more intuitively and clearly display the characteristics of the diseased brain areas. With this technology as a key examination for leukoaraiosis patients, its images and detection signals can all provide clinical basis for patients. Moreover, thanks to its strong tissue resolution ability, it can also provide clear imaging analysis on the tissues around the diseased brain area [21]. Therefore, the technology receives increasingly wider application in the field of physiotherapy. Treatment plan will be proposed after the examination of leukoaraiosis patients through magnetic resonance diffusion tensor imaging (MR-DTI), and patients should exactly follow the plan in post-treatment rehabilitation. Meanwhile, routine nursing and psychological intervention should be implemented [22-23]. First, it is necessary to understand and summarize the patient's current psychology, formulate the best treatment plan for the patient, keep the patient optimistic and positive, and create comfortable environment that facilitates recovery most; second, there is need to fully understand the patient's family, so that the family members have a better understanding of the patient's symptoms, pay attention to the patient's psychological changes in time, and relieve the negative emotions-induced factors unfavorable for rehabilitation [24]. At the same time, it is advisable to encourage patients to take various exercises and develop good living habits, which plays an important role in promoting patients' post-treatment rehabilitation [25]. Based on the above studies, as well as our study, we can indicate that diagnosis of MRI diffusion tensor imaging for leukoaraiosis can be possible with scientific theoretical basis to formulate corresponding treatment plans and promote rapid recovery, which also lays a solid foundation for further study of subsequent clinical trials of the disease [21].

We suggest to evaluate the rate of amplitude lowfrequency fluctuation along with the ReHo in further studies.

\section{Conflict of Interest}

The authors have no conflict of interest to declare.

\section{References}

1. GEBEILY S., FARES Y., KORDAHI M. et al. Cerebral white matter hyperintensities (WMH): an analysis of cerebrovascular risk factors in Lebanon, Int J Neurosci, 2014, 124(11), 799-805.

2. JIN B.X., FU W.J., QIAN X.G. et al. Clinical efficacy of different orthogonal design-based scalp acupuncture schemes on cerebral palsy, Chinese Journal of Rehabilitation Medicine, 2015, 30(6), 591-593.

3. ZHANG Z., REN W., SHAO B. et al. Leukoaraiosis is associated with worse short-term functional and cognitive recovery after minor stroke, Med Chir (Tokyo), 2017, 57(3), 136-143.

4. SI Q., ZHANG H., WANG X.M. et al. Application of magnetic resonance diffusion tensor imaging (DTI) in the diagnosis of central nervous system diseases, Journal of Practical Medical Imaging, 2011, 12(1), 4-7.

5. BOLANDZADEH N., DAVIS J.C., TAM R. et al. The association between cognitive function and white matter lesion location in older adults: a systematic review, BMC Neurol, 2012, 30(12), 126.

6. YU X., WANG G., ZHAN J. et al. Risk factors of pure leukoaraiosis and the association with preclinical carotid atherosclerosis, Atherosclerosis, 2018, 275(8), 328-332.

7. ZHANG A.J., WANG S.Z., WANG J.H. Regression analysis of risk factors for leukoaraiosis, Chinese Journal of Rehabilitation Theory and Practice, 2012, 18(11), 1069-1070.

8. FU H.X., ZHANG J., SUN Y.J. et al. The effect of elderly leukoaraiosis complicated with hypertension on patients' cognitive function, Journal of Apoplexy and Nervous Diseases, 2016, 33(1), 42-45.

9. BEAN J. Rey auditory verbal learning test, Rey AVLT. Encyclopedia of clinical neuropsychology. 2011:2174-5.

10. SCARPINA F, TAGINI S. The stroop color and word test. Frontiers in psychology. 2017 Apr 12; 8:557.

11. ALLAN C.L., ZSOLDOS E., FILIPPINI N. et al. Lifetime hypertension as predictor of brain structure in older adults: cohortstudy with a 28-year follow-up, Br J Psychiatry, 2015, 206(4), 308-315.

12. LIN Q., HUANG W.Q., MA Q.L. et al. Incidence and risk factors of leukoaraiosis from 4683 hospitalized patients: A cross-sectional study, Medicine (Baltimore), 2017, 96(39), e7682.

13. ZHANG H., WANG Y., GENG Z.J. et al. Diagnostic value of ultra-high-field magnetic resonance diffusion tensor imaging for leukoaraiosis, Chinese Journal of Geriatric Heart Brain and Vessel Diseases, 2012, 14(10), 1028-1031.

14. KIM T.W., KIM Y.H., KIM K.H. et al. White matter hyperintensities and cognitive dysfunction in patients with infratentorial stroke, Ann Rehabil Med, 2014, 38(5), 620-627.

15. WANG Y., ZHANG H., GENG Z.J. et al. Differential diagnosis value of high field magnetic resonance diffusion tensor imaging for simple leukoaraiosis and multiple sclerosis, Medical Recapitulate, 2015, 21(17), 3203-3206.

16. MOHD ZULKIFLY M.F., GHAZALI S.E., CHE DIN N. et al. A review of risk factors for cognitive impairment in stroke survivors, Scientific World Journal, 2016, 2016, 3456943. 
17. LI Q., HU F.Y., ZHAO L.Q. Research progress on leukoaraiosis and related cognitive impairment, Medical Innovation of China, 2015, 36, 150-153.

18. LIU Y., ZHANG A.J., GAO L.Z. et al. The correlation between multiple white matter microinfarctions and leukoaraiosis, Acta Academiae Medicinae Weifang, 2016, 38(6), 418-420.

19. TE M., ZHAO E., ZHENG X. et al. Leukoaraiosis with mild cognitive impairment, Neurol Res, 2015, 37(5), 410-414.

20. O'SULLIVAN M, MORRIS RG, HUCKSTEP B, JONES DK, WILLIAMS SC, MARKUS HS. Diffusion tensor MRI correlates with executive dysfunction in patients with ischaemic leukoaraiosis. Journal of Neurology, Neurosurgery \& Psychiatry. 2004 Mar 1; 75(3):441-7.

21. ZHAO DQ, WANG ZW, CHENG Y, YUAN Z, RENE F, LIU H, PLISS A, LUAN P. A DTI study of leukoaraiosis and the differential diagnosis between leukoaraiosis and acute lacunar infarction. CNS neuroscience \& therapeutics. 2019 Sep; 25(9):1064.

22. ZHU J, XU C, ZHANG X, QIAO L, WANG X, ZHANG X, YAN X, NI D, YU T, ZHANG G, LI Y.
Altered amplitude of low-frequency fluctuations and regional homogeneity in drug-resistant epilepsy patients with vagal nerve stimulators under different current intensity. CNS Neuroscience \& Therapeutics. 2020 Sep 23.

23. WEI L.F., WANG S.S., ZHENG Z.C. et al. Characteristics of MRI diffusion tensor imaging parameters of normal cervical spinal cord, Chinese Journal of Medical Imaging, 2016, 24(2), 91-95.

24. HAARMAN B.C.M, RIEMERSMA-VAN D.L.R.F, BURGER H. et al. Diffusion tensor imaging in euthymic bipolar disorder-a tract-based spatial statistics study, J Affect Disord, 2016, 203, 281-291.

25. LI Q., ZHAO L.Q, HU F.Y. Characteristics of cognitive impairment in leukoaraiosis patients and its resting-state functional magnetic resonance observation, National Medical Journal of China, 2017, 97(45), 56-58.

26. SHI K., WANG J., TANG Y., \& ZHONG S. (2020). Reliable asynchronous sampled-data filtering of $\mathrm{T}-\mathrm{S}$ fuzzy uncertain delayed neural networks with stochastic switched topologies. Fuzzy sets and systems, 381, 1-25. doi: 10.1016/j.fss.2018.11.01 